Mirian Carvalho de Souza

Ubirani Barros Otero

Liz Maria de Almeida

Silvana Rubano Barretto Turci

Valeska Carvalho Figueiredo

José de Azevedo Lozana

\section{Auto-avaliação de saúde e limitações físicas decorrentes de problemas de saúde}

\section{Self-rated health and physical disabilities due to heath problems}

\section{RESUMO}

OBJETIVO: Avaliar a autopercepção de saúde e a presença de limitações físicas devido a problemas de saúde.

MÉTODOS: Estudo transversal de base populacional realizado entre 2002 e 2005, em 18 capitais de estados do Brasil. Entrevistaram-se 26.424 moradores de 15 anos ou mais de idade em domicílios selecionados por amostra probabilística em dois estágios. Calcularam-se percentuais e intervalos de confiança considerando-se os efeitos do desenho do estudo.

RESULTADOS: Os resultados mostraram que as piores condições de saúde são referidas por mulheres, indivíduos com 50 anos ou mais e com menor grau de escolaridade. Os percentuais relacionados à percepção de saúde regular ou ruim foram maiores nas cidades das regiões Norte e Nordeste quando comparados aos das cidades das regiões Sul e Sudeste.

CONCLUSÕES: As piores condições de saúde das regiões Norte/Nordeste comparadas as das regiões Sul/Sudeste revelam um conjunto de fatores relacionados às desigualdades sociais, entre os quais o menor grau de escolaridade.

DESCRITORES: Condições de Saúde. Fatores Socioeconômicos. Estudos Transversais. Desigualdades em Saúde. 


\begin{abstract}
OBJECTIVE: To evaluate self-rated health and physical disabilities due to health problems.

METHODS: Population-based cross-sectional study carried out in 18 Brazilian cities between 2002 and 2005. There were interviewed 26,424 residents (aged 15 years or more) of households selected by a two-stage probabilistic sampling. Percentages and confidence intervals were calculated considering the study design effects.

RESULTS: Poorer self-rated health status and physical disabilities were found among women, those aged 50 years old or more and those with lower education. In addition, higher percentages of fair or poor self-rated health were found in cities in Northern and Northeastern Brazil compared to those in Southern and Southeastern Brazil.

CONCLUSIONS: Poorer health conditions in the North/Northeast compared to the South/Southeast regions reflect factors related to social inequalities, mainly lower education.

DESCRIPTORS: Health Status. Socioeconomic Factors. Cross-Sectional Studies. Health Inequalities.
\end{abstract}

\title{
INTRODUÇÃO
}

O Brasil deparou-se com declínio rápido e acentuado da fecundidade nas últimas décadas do século XX, que combinado com a queda da mortalidade, acarretou em processo de envelhecimento populacional. Esse cenário aponta sucesso em áreas de conhecimento como a saúde pública, e traz novos desafios pois a proporção de idosos no País está relacionada à demanda aos sistemas públicos de saúde e seguridade social. Sendo assim, o novo desafio da saúde pública é fazer com que a população viva de forma mais saudável e não apenas por mais tempo, apesar dos efeitos cumulativos associados ao processo de envelhecimento ou das doenças. ${ }^{1}$

O processo saúde-doença é dinâmico e reflete a ligação estrutural entre o corpo e a sociedade, os quais são cruciais para se estudar a percepção do estado de saúde. As desigualdades sociais podem ser examinadas por meio de avaliação dos indicadores de saúde de determinada população. Em geral, os mais utilizados são os relativos à mortalidade e a morbidade referida.?

Esses indicadores e a restrição de atividades rotineiras são importantes indicadores das condições de saúde quando a população é homogênea. ${ }^{6}$ São largamente utilizados para estudar a demanda por serviços de saúde, avaliar as condições de saúde populacionais e as políticas de saúde, e auxiliar a formulação de novas propostas de saúde pública. Além disso, o percentual de pessoas que define seu estado de saúde como regular ou ruim foi associado com a mortalidade em estudos longitudinais. ${ }^{13,16}$

Nos últimos anos, países desenvolvidos e em desenvolvimento têm experimentado crescimento da mortalidade por doenças crônicas que, em geral, afetam idosos com mais freqüência. Tais doenças causam limitações ou dificuldades que influenciam a habilidade relativa às atividades habituais, levando à diminuição da qualidade de vida e ao aumento dos custos da assistência a saúde. ${ }^{1}$

A ocorrência de fatores de risco associados a problemas de saúde varia segundo sexo e situação social., ${ }^{2,11,21}$ Os fatores que aumentam os riscos de doenças em mulheres são obesidade, sedentarismo, estresse, conseqüências de gestações repetidas, depressão e pressões ligadas aos papéis sociais. Entre os homens, comportamentos de risco - excesso de consumo de álcool, tabagismo, maior exposição a situações de violência, acidentes e riscos ocupacionais - são mais freqüentes e colaboram com aumento de riscos de problemas de saúde em longo prazo ${ }^{7,18} \mathrm{~A}$ presença desses e de outros fatores de riscos está associada ao desempenho dos papéis sociais dos indivíduos, o que afeta, diretamente tanto a qualidade de vida quanto a percepção do estado de saúde..,9

No Brasil, os resultados do Suplemento Saúde da Pesquisa Nacional por Amostra de Domicílios (PNAD) realizada em 2003 indicam que, aproximadamente, 25,6\% da população de 14 anos ou mais (22,5\% dos homens e $28,5 \%$ das mulheres) auto-avaliaram seu estado de saúde como regular, ruim ou muito ruim; $;^{5} 6,7 \%$ referiram restrição de atividades rotineiras por motivo de saúde nas duas semanas que antecederam a entrevista.

O objetivo do presente estudo foi avaliar a percepção de saúde e a presença de limitações físicas decorrentes de problemas de saúde na população. 
Tabela 1. Percentual de indivíduos que consideraram o seu próprio estado de saúde regular ou ruim, segundo cidade e sexo. Brasil, 2002-2005.

\begin{tabular}{|c|c|c|c|c|c|c|}
\hline \multirow{3}{*}{ Região/Cidade } & \multirow{2}{*}{\multicolumn{2}{|c|}{ Total }} & \multicolumn{4}{|c|}{ Sexo } \\
\hline & & & \multicolumn{2}{|c|}{ Masculino } & \multicolumn{2}{|c|}{ Feminino } \\
\hline & $\%$ & IC 95\% & $\%$ & IC 95\% & $\%$ & IC 95\% \\
\hline \multicolumn{7}{|l|}{ Região Norte } \\
\hline Manaus & 31,0 & 28,$2 ; 33,8$ & 22,7 & 19,$5 ; 26,0$ & 37,8 & 34,$1 ; 41,4$ \\
\hline Belém & 36,0 & 32,$5 ; 39,4$ & 31,5 & 27,$2 ; 35,8$ & 39,3 & 35,$0 ; 43,5$ \\
\hline Palmas & 26,7 & 21,$8 ; 31,6$ & 23,2 & 18,$0 ; 28,3$ & 30,0 & 23,$8 ; 36,2$ \\
\hline \multicolumn{7}{|l|}{ Região Nordeste } \\
\hline São Luís & 34,8 & 31,$1 ; 38,6$ & 31,5 & 26,$8 ; 36,1$ & 37,4 & 33,$4 ; 41,5$ \\
\hline Fortaleza & 27,7 & 25,$2 ; 30,3$ & 22,8 & 19,$9 ; 25,7$ & 31,6 & 28,$2 ; 35,0$ \\
\hline Natal & 31,1 & 27,$1 ; 35,0$ & 25,1 & 20,$7 ; 29,5$ & 35,7 & 30,$7 ; 40,6$ \\
\hline João Pessoa & 26,7 & 22,$8 ; 30,6$ & 20,4 & 16,$2 ; 24,6$ & 31,0 & 26,$0 ; 36,1$ \\
\hline Recife & 32,4 & 28,$3 ; 36,4$ & 26,5 & 21,$5 ; 31,5$ & 36,8 & 31,$7 ; 41,9$ \\
\hline Aracaju & 32,8 & 28,$3 ; 37,3$ & 26,3 & 20,$5 ; 32,2$ & 37,5 & 32,$4 ; 42,6$ \\
\hline \multicolumn{7}{|l|}{ Região Sudeste } \\
\hline Belo Horizonte & 19,5 & 17,$0 ; 21,9$ & 16,2 & 13,$6 ; 18,9$ & 22,0 & 18,$8 ; 25,2$ \\
\hline Vitória & 23,4 & 18,$6 ; 28,1$ & 19,9 & 14,$4 ; 25,4$ & 26,3 & 20,$8 ; 31,9$ \\
\hline Rio de Janeiro & 25,6 & 23,$5 ; 27,7$ & 18,8 & 16,$2 ; 21,4$ & 30,3 & 27,$8 ; 32,9$ \\
\hline São Paulo & 24,5 & 21,$8 ; 27,1$ & 20,6 & 17,$4 ; 23,8$ & 27,4 & 23,$9 ; 30,9$ \\
\hline \multicolumn{7}{|l|}{ Região Sul } \\
\hline Curitiba & 19,7 & 17,$7 ; 21,7$ & 17,2 & 14,$7 ; 19,8$ & 21,7 & 19,$3 ; 24,1$ \\
\hline Florianópolis & 21,4 & 18,$3 ; 24,5$ & 17,7 & 13,$9 ; 21,5$ & 24,4 & 20,$5 ; 28,4$ \\
\hline Porto Alegre & 18,4 & 15,$9 ; 20,8$ & 16,3 & 13,$0 ; 19,7$ & 20,0 & 16,$8 ; 23,2$ \\
\hline \multicolumn{7}{|l|}{ Região Centro-Oeste } \\
\hline Campo Grande & 19,2 & 15,$2 ; 23,2$ & 19,5 & 14,$8 ; 24,2$ & 19,0 & 14,$4 ; 23,7$ \\
\hline Brasília & 29,0 & 25,$3 ; 32,7$ & 24,4 & 20,$9 ; 28,0$ & 32,5 & 27,$5 ; 37,5$ \\
\hline
\end{tabular}

\section{MÉTODOS}

Os dados são provenientes de um estudo transversal de base populacional realizado entre 2002 e 2005, denominado "Inquérito Domiciliar sobre Comportamentos de Risco e Morbidade Referida de Doenças e Agravos não Transmissíveis”.

A população-alvo consistiu de indivíduos com idade igual ou superior a 15 anos no momento da pesquisa, residentes em 18 capitais de estados: Manaus, Belém, Palmas, Aracaju, Fortaleza, João Pessoa, Natal, Recife, São Luís, Belo Horizonte, Rio de Janeiro, São Paulo, Vitória, Curitiba, Florianópolis, Porto Alegre, Brasília e Campo Grande. Apesar da seleção destas cidades ter sido afetada por fatores logísticos e operacionais, todas as macro-regiões do País estão representas por pelo menos duas cidades. Nas regiões Sul e Sudeste todas as capitais foram contempladas.

O modelo de amostragem adotado foi o de amostra autoponderada com dois estágios de seleção, sendo as unidades primárias os setores censitários e as unidades secundárias, os domicílios. A seleção desses setores foi feita de forma sistemática, com probabilidade de seleção proporcional ao número de domicílios que os mesmos possuíam por ocasião do censo demográfico. Uma seleção sistemática foi também utilizada com relação aos domicílios dentro dos setores escolhidos. Todos os moradores de 15 anos ou mais dos domicílios selecionados foram convidados a participar da pesquisa.

O instrumento de coleta de dados foi um questionário multidimensional, aplicado por entrevistadoras treinadas. Para a construção desse questionário foram realizadas oficinas com especialistas e foram revisados os instrumentos nacionais e internacionais amplamente utilizados para avaliar dados socioeconômicos, demográficos, morbidade referida, estilo e qualidade de vida, capacidade funcional, acesso a serviços de saúde, acidentes de trânsito e violência entre parceiros.

\footnotetext{
a Instituto Nacional de Câncer. Inquérito domiciliar sobre comportamentos de risco e morbidade referida de doenças e agravos não transmissíveis. Rio de Janeiro; 2003. [citado 2008 abr 9] Disponível em: http://www.inca.gov.br/inquerito
} 
Tabela 2. Percentual de indivíduos que referiram ter limitação ou dificuldade para fazer as suas atividades habituais por causa de algum problema de saúde ou incapacitação, segundo cidade e sexo. Brasil, 2002-2005.

\begin{tabular}{|c|c|c|c|c|c|c|}
\hline \multirow{3}{*}{ Região/Cidade } & \multirow{2}{*}{\multicolumn{2}{|c|}{ Total }} & \multicolumn{4}{|c|}{ Sexo } \\
\hline & & & \multicolumn{2}{|c|}{ Masculino } & \multicolumn{2}{|c|}{ Feminino } \\
\hline & $\%$ & IC 95\% & $\%$ & IC $95 \%$ & $\%$ & IC 95\% \\
\hline \multicolumn{7}{|l|}{ Região Norte } \\
\hline Manaus & 13,7 & 11,$8 ; 15,6$ & 11,5 & 8,$8 ; 14,2$ & 15,5 & 13,$0 ; 18,0$ \\
\hline Belém & 18,1 & 15,$3 ; 20,8$ & 12,4 & 9,$9 ; 14,8$ & 22,3 & 18,$9 ; 25,8$ \\
\hline Palmas & 15,0 & 11,$7 ; 18,2$ & 13,1 & 9,$6 ; 16,6$ & 16,7 & 12,$4 ; 21,1$ \\
\hline \multicolumn{7}{|l|}{ Região Nordeste } \\
\hline São Luís & 12,2 & 10,$5 ; 13,9$ & 8,0 & 5,$9 ; 10,0$ & 15,4 & 12,$9 ; 17,9$ \\
\hline Fortaleza & 16,2 & 14,$2 ; 18,1$ & 10,2 & 8,$2 ; 12,2$ & 20,9 & 18,$2 ; 23,5$ \\
\hline Natal & 15,7 & 13,$0 ; 18,4$ & 9,9 & 6,$7 ; 13,2$ & 20,1 & 16,$0 ; 24,2$ \\
\hline João Pessoa & 7,7 & 5,$0 ; 10,5$ & 5,5 & 3,$2 ; 7,8$ & 9,2 & 5,$6 ; 12,9$ \\
\hline Recife & 15,3 & 13,$1 ; 17,6$ & 10,4 & 7,$5 ; 13,3$ & 19,1 & 16,$1 ; 22,1$ \\
\hline Aracaju & 14,6 & 11,$2 ; 18,1$ & 10,2 & 6,$8 ; 13,6$ & 17,8 & 13,$2 ; 22,5$ \\
\hline \multicolumn{7}{|l|}{ Região Sudeste } \\
\hline Belo Horizonte & 12,3 & 10,$5 ; 14,2$ & 7,8 & 6,$1 ; 9,6$ & 15,8 & 13,$1 ; 18,5$ \\
\hline Vitória & 11,5 & 9,$1 ; 13,9$ & 6,8 & 3,$6 ; 10,0$ & 15,5 & 12,$1 ; 18,8$ \\
\hline Rio de Janeiro & 11,2 & 10,$1 ; 12,3$ & 8,0 & 6,$4 ; 9,7$ & 13,4 & 11,$9 ; 14,9$ \\
\hline São Paulo & 12,2 & 10,$2 ; 14,3$ & 7,5 & 5,$2 ; 9,7$ & 15,9 & 12,$9 ; 18,9$ \\
\hline \multicolumn{7}{|l|}{ Região Sul } \\
\hline Curitiba & 12,7 & 11,$2 ; 14,1$ & 10,2 & 8,$3 ; 12,0$ & 14,7 & 12,$7 ; 16,7$ \\
\hline Florianópolis & 17,7 & 14,$3 ; 21,2$ & 10,6 & 7,$4 ; 13,7$ & 23,5 & 18,$3 ; 28,7$ \\
\hline Porto Alegre & 18,9 & 16,$4 ; 21,4$ & 14,7 & 11,$3 ; 18,1$ & 22,3 & 19,$3 ; 25,3$ \\
\hline \multicolumn{7}{|l|}{ Região Centro-Oeste } \\
\hline Campo Grande & 15,6 & 12,$6 ; 18,6$ & 13,3 & 9,$4 ; 17,3$ & 17,5 & 13,$2 ; 21,8$ \\
\hline Brasília & 14,5 & 12,$6 ; 16,4$ & 9,4 & 7,$3 ; 11,4$ & 18,4 & 15,$6 ; 21,2$ \\
\hline
\end{tabular}

Para avaliar a percepção de saúde, utilizou-se a pergunta: "De um modo geral, em comparação com pessoas da sua idade, como o(a) sr.(a) considera o seu próprio estado de saúde?”, que mede a auto-avaliação do estado de saúde em escala de cinco categorias (excelente, muito bom, bom, regular ou ruim). Esta pergunta tem sido utilizada freqüentemente em estudos internacionais, ${ }^{4,17}$ tendo sido aplicada no Suplemento Saúde da PNAD realizada em 2003. ${ }^{\text {,a }}$ Nas 18 cidades, de um total de 25.123.974 residentes com 15 anos de idade ou mais, 26.424 foram entrevistados e destes mais de 99,9\% responderam ao questionário. A cidade com menor número de participantes foi Campo Grande (697 entrevistados, 308 do sexo masculino e 389 do sexo feminino) e a cidade com maior número de participantes foi Rio de Janeiro (2.692 entrevistados, 1.096 do sexo masculino e 1.596 do sexo feminino). Com base nas respostas, foi gerado o percentual de indivíduos que em comparação com pessoas de sua idade, consideraram o seu próprio estado de saúde regular ou ruim.
A presença de limitações físicas foi avaliada, utilizando-se a pergunta: “O(a) sr.(a) tem alguma limitação ou dificuldade para fazer as suas atividades habituais por causa de algum problema de saúde?”, semelhante à utilizada em outro estudo internacional ${ }^{4}$ e no Suplemento

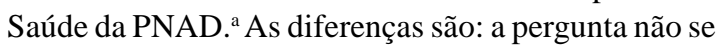
restringiu a uma incapacidade por um período de duas semanas como na PNAD, mas sim a incapacidade de longa duração; e foi realizada de forma direta (autoavaliação) enquanto na PNAD havia a possibilidade de um entrevistado avaliar as condições de saúde dos demais moradores do domicílio. Com base nas respostas foi gerado o percentual de indivíduos que referiram ter limitação ou dificuldade para fazer as suas atividades habituais por causa de algum problema de saúde ou incapacitação.

Além desses percentuais, foram calculados intervalos com 95\% de confiança para as estimativas segundo capital e as co-variáveis: sexo, faixas etárias (15 a

a Instituto Brasileiro de Geografia e Estatística. Pesquisa Nacional por Amostra de Domicílios 2003. Rio de Janeiro; 2003. [citado 2008 abr 9]. Disponível em http://www.ibge.gov.br/home/estatistica/populacao/trabalhoerendimento/pnad2003/default.shtm. 
Tabela 3. Percentual de indivíduos que consideraram o seu próprio estado de saúde regular ou ruim, segundo cidade e faixas etárias. Brasil, 2002-2005.

\begin{tabular}{|c|c|c|c|c|c|c|}
\hline \multirow{3}{*}{ Região/Cidade } & \multicolumn{6}{|c|}{ Faixa etária } \\
\hline & \multicolumn{2}{|c|}{15 a 24 anos } & \multicolumn{2}{|c|}{25 a 49 anos } & \multicolumn{2}{|c|}{50 anos e mais } \\
\hline & $\%$ & IC 95\% & $\%$ & IC 95\% & $\%$ & IC 95\% \\
\hline \multicolumn{7}{|l|}{ Região Norte } \\
\hline Manaus & 24,7 & 20,$9 ; 28,5$ & 29,3 & 25,$2 ; 33,3$ & 49,6 & 42,$5 ; 56,8$ \\
\hline Belém & 32,1 & 27,$3 ; 36,9$ & 34,6 & 30,$2 ; 38,9$ & 45,8 & 39,$3 ; 52,3$ \\
\hline Palmas & 23,9 & 17,$1 ; 30,6$ & 25,8 & 20,$6 ; 31,0$ & 41,5 & 30,$9 ; 52,1$ \\
\hline \multicolumn{7}{|l|}{ Região Nordeste } \\
\hline São Luís & 24,8 & 20,$5 ; 29,1$ & 35,9 & 31,$4 ; 40,4$ & 52,1 & 44,$5 ; 59,8$ \\
\hline Fortaleza & 21,4 & 18,$0 ; 24,8$ & 24,7 & 21,$6 ; 27,7$ & 42,5 & 37,$4 ; 47,7$ \\
\hline Natal & 18,3 & 12,$9 ; 23,8$ & 29,8 & 25,$2 ; 34,4$ & 51,7 & 44,$6 ; 58,9$ \\
\hline João Pessoa & 14,4 & 10,$0 ; 18,9$ & 25,8 & 20,$3 ; 31,4$ & 43,4 & 36,$1 ; 50,8$ \\
\hline Recife & 24,6 & 18,$3 ; 30,9$ & 30,7 & 25,$9 ; 35,5$ & 42,7 & 35,$1 ; 50,3$ \\
\hline Aracaju & 27,4 & 21,$1 ; 33,8$ & 30,8 & 25,$4 ; 36,3$ & 46,1 & 37,$4 ; 54,7$ \\
\hline \multicolumn{7}{|l|}{ Região Sudeste } \\
\hline Belo Horizonte & 13,7 & 10,$2 ; 17,1$ & 18,6 & 15,$8 ; 21,5$ & 28,0 & 22,$9 ; 33,0$ \\
\hline Vitória & 17,6 & 12,$2 ; 23,0$ & 22,8 & 16,$9 ; 28,7$ & 30,5 & 22,$2 ; 38,8$ \\
\hline Rio de Janeiro & 17,1 & 13,$5 ; 20,8$ & 23,0 & 20,$2 ; 25,8$ & 34,9 & 31,$3 ; 38,6$ \\
\hline São Paulo & 16,0 & 11,$8 ; 20,1$ & 24,0 & 20,$0 ; 28,0$ & 35,8 & 30,$1 ; 41,5$ \\
\hline \multicolumn{7}{|l|}{ Região Sul } \\
\hline Curitiba & 13,7 & 10,$5 ; 16,9$ & 16,8 & 14,$5 ; 19,0$ & 33,6 & 28,$3 ; 38,9$ \\
\hline Florianópolis & 13,4 & 8,$9 ; 17,9$ & 17,9 & 13,$6 ; 22,3$ & 33,7 & 27,$7 ; 39,8$ \\
\hline Porto Alegre & 10,9 & 7,$2 ; 14,7$ & 18,6 & 15,$1 ; 22,0$ & 23,8 & 19,$0 ; 28,7$ \\
\hline \multicolumn{7}{|l|}{ Região Centro-Oeste } \\
\hline Campo Grande & 11,7 & 7,$8 ; 15,5$ & 16,3 & 10,$8 ; 21,7$ & 35,2 & 26,$5 ; 44,0$ \\
\hline Brasília & 21,8 & 16,$3 ; 27,3$ & 26,9 & 23,$2 ; 30,7$ & 47,2 & 39,$6 ; 54,8$ \\
\hline
\end{tabular}

24 anos; 25 a 49 anos; 50 anos e mais) e faixas de escolaridade (ensino fundamental incompleto; ensino fundamental completo ou mais). A significância estatística das estimativas foi avaliada comparando-se os limites dos intervalos de confiança. Todas as estatísticas foram calculadas considerando-se o efeito do desenho amostral no software Stata 8.0.

Todos participantes assinaram um termo de consentimento livre e esclarecido. No caso de participantes menores de 18 anos, o responsável (pais ou guardião) assinou o termo. O estudo foi realizado segundo os critérios da Comissão Nacional de Ética em Pesquisa.

\section{RESULTADOS}

Na Tabela 1 observa-se que a percepção de saúde considerada regular ou ruim apresenta valores que variaram entre 18,4\% (Porto Alegre) e 36,0\% (Belém) e que os percentuais encontrados são homogêneos por região. Quando os limites dos intervalos de variação desse percentual são comparados entre as regiões Sul/Sudeste
(18,4\% a $25,6 \%$ ) e Norte/Nordeste (26,7\% a 36,0\%), verifica-se que não coincidem. Assim, há dois grupos distintos de cidades. O mesmo comportamento não foi observado na avaliação por sexo.

Com exceção de Campo Grande, de forma geral, as mulheres referiram com mais freqüência do que os homens uma percepção de saúde regular ou ruim, mas não foi observada diferença estatisticamente significativa quando avaliados os intervalos de confiança entre os sexos em Belém, Palmas, São Luís, Belo Horizonte, Vitória e nas capitais estudadas das regiões Sul e Centro-Oeste (Tabela 1).

Em relação à presença de limitações físicas devido a problemas de saúde, o mesmo padrão é observado na Tabela 2, e a maior diferença entre os sexos foi encontrada em Vitória (6,8\% para homens e 15,5\% para mulheres). Desconsiderando-se a estratificação por sexo, esse percentual variou de 7,7\%, em João Pessoa a 18,9\%, em Porto Alegre. Além de João Pessoa e São Luís, as capitais da região Sudeste foram as que apresentam os menores valores desse percentual. 
Tabela 4. Percentual de indivíduos que referiram ter limitação ou dificuldade para fazer as suas atividades habituais por causa de algum problema de saúde ou incapacitação, segundo cidade e faixas etárias. Brasil, 2002-2005.

\begin{tabular}{|c|c|c|c|c|c|c|}
\hline \multirow{3}{*}{ Região/Cidade } & \multicolumn{6}{|c|}{ Faixa etária } \\
\hline & \multicolumn{2}{|c|}{15 a 24 anos } & \multicolumn{2}{|c|}{25 a 49 anos } & \multicolumn{2}{|c|}{50 anos e mais } \\
\hline & $\%$ & IC 95\% & $\%$ & IC 95\% & $\%$ & IC 95\% \\
\hline \multicolumn{7}{|l|}{ Região Norte } \\
\hline Manaus & 10,5 & 7,$7 ; 13,2$ & 12,5 & 10,$6 ; 14,5$ & 24,1 & 17,$0 ; 31,1$ \\
\hline Belém & 12,1 & 8,$6 ; 15,5$ & 17,4 & 14,$0 ; 20,8$ & 29,5 & 23,$6 ; 35,4$ \\
\hline Palmas & 10,9 & 7,$5 ; 14,3$ & 14,4 & 10,$9 ; 18,0$ & 32,3 & 24,$1 ; 40,5$ \\
\hline \multicolumn{7}{|l|}{ Região Nordeste } \\
\hline São Luís & 7,1 & 5,$4 ; 8,9$ & 12,3 & 10,$1 ; 14,5$ & 22,0 & 16,$2 ; 27,8$ \\
\hline Fortaleza & 7,4 & 5,$2 ; 9,7$ & 16,5 & 14,$1 ; 18,9$ & 26,6 & 22,$9 ; 30,3$ \\
\hline Natal & 7,5 & 3,$8 ; 11,2$ & 14,1 & 10,$5 ; 17,7$ & 30,5 & 24,$2 ; 36,7$ \\
\hline João Pessoa & 2,2 & 0,$4 ; 4,0$ & 6,8 & 3,$6 ; 9,9$ & 16,5 & 10,$1 ; 22,9$ \\
\hline Recife & 6,9 & 3,$1 ; 10,6$ & 11,5 & 8,$8 ; 14,2$ & 30,3 & 24,$1 ; 36,5$ \\
\hline Aracaju & 7,6 & 4,$3 ; 11,0$ & 15,4 & 11,$3 ; 19,5$ & 23,0 & 15,$8 ; 30,3$ \\
\hline \multicolumn{7}{|l|}{ Região Sudeste } \\
\hline Belo Horizonte & 4,6 & 2,$8 ; 6,5$ & 10,5 & 8,$3 ; 12,7$ & 25,2 & 20,$5 ; 29,9$ \\
\hline Vitória & 7,0 & 3,$6 ; 10,4$ & 11,4 & 7,$8 ; 15,1$ & 16,3 & 11,$0 ; 21,6$ \\
\hline Rio de Janeiro & 4,1 & 2,$5 ; 5,7$ & 8,8 & 7,$3 ; 10,4$ & 19,3 & 16,$9 ; 21,8$ \\
\hline São Paulo & 5,5 & 2,$9 ; 8,2$ & 11,3 & 8,$6 ; 13,9$ & 22,5 & 16,$9 ; 28,1$ \\
\hline \multicolumn{7}{|l|}{ Região Sul } \\
\hline Curitiba & 7,3 & 5,$1 ; 9,5$ & 10,0 & 8,$3 ; 11,7$ & 25,2 & 21,$7 ; 28,6$ \\
\hline Florianópolis & 8,2 & 4,$1 ; 12,4$ & 15,7 & 11,$3 ; 20,1$ & 28,8 & 23,$0 ; 34,6$ \\
\hline Porto Alegre & 10,6 & 7,$4 ; 13,9$ & 16,5 & 13,$4 ; 19,5$ & 29,3 & 23,$8 ; 34,8$ \\
\hline \multicolumn{7}{|l|}{ Região Centro-Oeste } \\
\hline Campo Grande & 11,2 & 6,$9 ; 15,5$ & 12,7 & 8,$6 ; 16,7$ & 27,7 & 19,$9 ; 35,4$ \\
\hline Brasília & 6,9 & 4,$6 ; 9,1$ & 14,9 & 12,$2 ; 17,6$ & 26,1 & 21,$6 ; 30,5$ \\
\hline
\end{tabular}

As Tabelas 3 e 4 mostram que, conforme o esperado, quanto mais elevada a faixa etária, maiores são os percentuais de percepção de saúde regular ou ruim e de presença de limitações físicas. Maiores valores da percepção do estado de saúde regular ou ruim foram encontrados em todas as faixas etárias, nas capitais das regiões Norte e Nordeste.

Quando analisados por escolaridade (Tabela 5), os resultados indicam que, entre os indivíduos que completaram o ensino fundamental, a percepção de saúde regular ou ruim e a presença de limitações físicas foi menor quando comparada aos indivíduos que não completaram o ensino fundamental.

Para percepção de saúde, em todas as capitais estudadas, a comparação dos limites de confiança por faixas de escolaridade indica que as diferenças são estatisticamente significativas (Tabela 5). Para presença de limitações físicas, em Manaus, Belém, Aracaju e Vitória as diferenças entre grupos de escolaridade não foram estatisticamente significativas e em João Pessoa encontrou-se maior diferença entre os grupos estudados (Tabela 5).

\section{DISCUSSÃO}

Embora a percepção do estado de saúde possa variar de acordo com as experiências individuais, esse aspecto não foi avaliado no presente estudo, o que se constitui uma limitação. Outra limitação refere-se à seleção dos municípios. Como foram estudadas apenas capitais, os dados não podem ser generalizados como representativos do Brasil. Quanto à composição das áreas estudadas, somente aglomerados urbanos foram avaliados, portanto os dados não refletem a realidade de áreas rurais.

Apesar dessas limitações, as informações desagregadas por capital poderão ser úteis aos gestores locais para o planejamento em saúde.

No presente estudo, a maioria dos resultados relativos à percepção de saúde e presença de limitações físicas segundo sexo, faixa etária e escolaridade foram semelhantes aos de outros estudos realizados no Brasil ${ }^{5,16,20}$ e no exterior. ${ }^{8,12,15}$

A comparação dos resultados da presente pesquisa com os do Suplemento Saúde da PNAD deve ser cautelosa e 
Tabela 5. Percentual de indivíduos que consideraram o seu próprio estado de saúde regular ou ruim e de indivíduos que referiram ter limitação ou dificuldade para fazer as suas atividades habituais por causa de algum problema de saúde ou incapacitação, segundo cidade e escolaridade. Brasil, 2002-2005.

\begin{tabular}{|c|c|c|c|c|c|c|c|c|}
\hline \multirow{4}{*}{ Região/cidade } & \multirow{2}{*}{\multicolumn{4}{|c|}{$\begin{array}{l}\text { Estado de saúde referido como regular ou ruim } \\
\text { Ensino fundamental }\end{array}$}} & \multirow{2}{*}{\multicolumn{4}{|c|}{$\begin{array}{l}\text { Limitação ou dificuldade referida } \\
\text { Ensino fundamental }\end{array}$}} \\
\hline & & & & & & & & \\
\hline & \multicolumn{2}{|c|}{ Incompleto } & \multicolumn{2}{|c|}{ Completo e mais } & \multicolumn{2}{|c|}{ Incompleto } & \multicolumn{2}{|c|}{ Completo e mais } \\
\hline & $\%$ & IC 95\% & $\%$ & IC 95\% & $\%$ & IC $95 \%$ & $\%$ & IC 95\% \\
\hline \multicolumn{9}{|l|}{ Região Norte } \\
\hline Manaus & 42 & 38,$5 ; 45,3$ & 23 & 19,$4 ; 25,7$ & 16 & 12,$7 ; 19,1$ & 12 & 9,$8 ; 14,7$ \\
\hline Belém & 43 & 38,$0 ; 47,7$ & 31 & 27,$5 ; 35,1$ & 22 & 17,$7 ; 26,5$ & 15 & 12,$2 ; 18,3$ \\
\hline Palmas & 40 & 35,$0 ; 45,4$ & 20 & 15,$2 ; 25,6$ & 22 & 16,$9 ; 27,3$ & 12 & 8,$9 ; 14,5$ \\
\hline \multicolumn{9}{|l|}{ Região Nordeste } \\
\hline São Luís & 48 & 42,$6 ; 52,9$ & 29 & 25,$5 ; 32,1$ & 18 & 15,$0 ; 21,6$ & 9,2 & 7,$5 ; 10,9$ \\
\hline Fortaleza & 39 & 35,$5 ; 42,3$ & 19 & 17,$1 ; 21,5$ & 20 & 17,$0 ; 23,2$ & 13 & 11,$1 ; 14,7$ \\
\hline Natal & 41 & 36,$2 ; 46,5$ & 21 & 16,$6 ; 25,4$ & 19 & 15,$0 ; 23,1$ & 11 & 8,$1 ; 14,4$ \\
\hline João Pessoa & 37 & 32,$5 ; 40,7$ & 18 & 14,$3 ; 22,1$ & 12 & 7,$1 ; 16,0$ & 4,4 & 2,$3 ; 6,4$ \\
\hline Recife & 44 & 39,$3 ; 48,3$ & 23 & 18,$3 ; 27,1$ & 22 & 17,$6 ; 25,5$ & 11 & 8,$1 ; 13,3$ \\
\hline Aracaju & 43 & 37,$6 ; 47,6$ & 25 & 20,$3 ; 30,2$ & 18 & 12,$4 ; 23,1$ & 12 & 8,$9 ; 15,9$ \\
\hline \multicolumn{9}{|l|}{ Região Sudeste } \\
\hline Belo Horizonte & 30 & 26,$0 ; 33,7$ & 12 & 10,$1 ; 14,6$ & 18 & 15,$2 ; 20,3$ & 8,4 & 6,$7 ; 10,1$ \\
\hline Vitória & 43 & 35,$1 ; 51,7$ & 16 & 12,$1 ; 20,4$ & 16 & 10,$9 ; 20,7$ & 9,9 & 7,$2 ; 12,7$ \\
\hline Rio de Janeiro & 40 & 36,$2 ; 42,9$ & 20 & 17,$5 ; 21,5$ & 15 & 12,$5 ; 17,1$ & 9,3 & 8,$0 ; 10,5$ \\
\hline São Paulo & 37 & 32,$2 ; 41,3$ & 17 & 14,$1 ; 19,7$ & 16 & 12,$6 ; 19,7$ & 10 & 7,$8 ; 12,1$ \\
\hline \multicolumn{9}{|l|}{ Região Sul } \\
\hline Curitiba & 31 & 27,$1 ; 35,0$ & 14 & 12,$1 ; 15,3$ & 17 & 14,$3 ; 19,7$ & 10 & 8,$6 ; 11,9$ \\
\hline Florianópolis & 37 & 30,$5 ; 43,0$ & 14 & 10,$9 ; 16,7$ & 25 & 19,$2 ; 30,8$ & 14 & 9,$8 ; 17,4$ \\
\hline Porto Alegre & 33 & 29,$4 ; 37,4$ & 12 & 9,$9 ; 13,9$ & 30 & 24,$5 ; 34,6$ & 14 & 12,$0 ; 16,8$ \\
\hline \multicolumn{9}{|l|}{ Região Centro-Oeste } \\
\hline Campo Grande & 30 & 23,$4 ; 36,0$ & 12 & 8,$2 ; 14,8$ & 22 & 16,$6 ; 27,1$ & 11 & 7,$6 ; 14,9$ \\
\hline Brasília & 45 & 40,$5 ; 49,0$ & 20 & 16,$8 ; 23,6$ & 18 & 14,$9 ; 20,4$ & 12 & 9,$8 ; 14,7$ \\
\hline
\end{tabular}

considerar as diferenças metodológicas, pois na PNAD, o entrevistado podia avaliar as condições de saúde dos demais moradores do domicílio.

À semelhança de outros achados, como os da Pesquisa Mundial de Saúde no Brasil (PMSB) ${ }^{20}$ realizada em 2003 e os da PNAD, ${ }^{a}$ que encontraram que as piores auto-avaliações foram relatadas por mulheres e os mais idosos, a presente pesquisa encontrou diferenças estatisticamente significativas em algumas cidades estudadas. Apenas em Campo Grande, os homens referiram piores condições com mais freqüência, quando comparados as mulheres.

Estudos sobre diferenças em relação à saúde segundo sexo em sociedades industrializadas indicam que, embora vivam mais do que os homens, as mulheres referem mais morbidade e problemas psicológicos e utilizam mais os serviços de saúde.,211,12,16 Estudos norte-americanos revelam que os homens sofrem de mais doenças crônicas fatais e referem mais restrições de atividades e incapacidade de longa duração devido a problemas crônicos de saúde.,21 Segundo dados do National Health Interview Survey (NHIS), nos Estados Unidos, o percentual de indivíduos que auto-avaliaram seu estado de saúde como regular ou ruim era de 9,5\% em 1987 (9,0\% entre homens e 9,9\% entre mulheres) e aumentou para $12,2 \%$ (11,9\% entre homens e $12,6 \%$ entre mulheres) em 2002. ${ }^{10}$ Limitações para execução de atividades habituais foram referidas por $12,6 \%$ dos entrevistados, dos quais $12,7 \%$ entre os homens e $12,8 \%$ entre as mulheres. ${ }^{19}$

Os resultados do NHIS de 1996 indicam que, em geral, as condições de saúde referidas no Brasil são piores do que as declaradas pelos estadunidenses, independentemente dos estratos de avaliação (sexo ou faixa etária). ${ }^{14}$

a Instituto Brasileiro de Geografia e Estatística. Pesquisa Nacional por Amostra de Domicílios 2003. Rio de Janeiro; 2003. [citado 2008 abr 9]. Disponível em http://www.ibge.gov.br/home/estatistica/populacao/trabalhoerendimento/pnad2003/default.shtm 
A homogeneidade interna dos percentuais encontrados nos grupos Norte/Nordeste e Sul/Sudeste e a heterogeneidade entre eles reflete diferenças regionais, onde persistem as desigualdades sociais. ${ }^{\mathrm{a}}$

No presente artigo, a escolaridade foi utilizada como proxy da condição socioeconômica individual e quanto menor a escolaridade, maior foi o percentual de indivíduos que referiram piores condições de saúde em todas as cidades estudadas. Esse comportamento é reflexo de diversos fatores, incluindo-se a condição socioeconômica, estilo de vida, exposições ambientais diferenciadas e desigualdade social. Nos Estados Unidos, o mesmo comportamento foi observado nos últimos anos, conforme publicado nos relatórios do National Center for Health Statistics. ${ }^{15}$

A maior prevalência de limitações físicas em uma cidade ou região comparada a outra refletiu um conjunto de fatores, entre os quais a estrutura etária da população.
Os achados do presente estudo confirmam o esperado, ou seja, indivíduos com mais idade referem com mais freqüência que o seu estado de saúde é regular ou ruim ao apresentar condições limitantes ou incapacitantes do seu estado de saúde. Dessa forma, os resultados apontam a necessidade de enfrentamento de um problema de saúde pública que tende a aumentar, devido ao envelhecimento da população.

A coleta e a disseminação sistemática de informações relacionadas à percepção de saúde e à presença de limitações físicas deve ser estimulada no Brasil, pois essas informações podem subsidiar a identificação e a formulação de novas propostas visando à melhoria das condições de vida da população.

\section{AGRADECIMENTOS}

À Fundação Instituto Brasileiro de Geografia e Estatística pelo apoio na organização da coleta de dados.

\footnotetext{
a Ministério da Saúde. Saúde no Brasil: contribuições para a Agenda de Prioridades de Pesquisa. Brasília; 2004. (Série B. Textos Básicos de Saúde).
} 


\section{REFERÊNCIAS}

1. Alves LC, Rodrigues RN. Determinantes da autopercepção de saúde entre idosos do Município de São Paulo, Brasil. Rev Panam Salud Publica. 2005;17(5/6):333-341.

2. Bird CE, Rieker PP. Gender matters: an integrated model for understanding men's and women's health. Soc Sci Med. 1999;48(6):745-55. doi:10.1016/S02779536(98)00402-X

3. Boltanski L. As classes sociais e o corpo. 3. ed. Rio de Janeiro: Graal; 1989.

4. Centers for Disease Control and Prevention. Behavioral risk factor surveillance system survey questionnaire. Atlanta (GA); 2006.

5. Dachs JNW, Santos APR. Auto-avaliação do estado de saúde no Brasil: análise dos dados da PNAD/2003. Cienc Saude Coletiva. 2006;11(4):887-94. doi:10.1590/S1413-81232006000400012

6. de Bruin A, Picavet HSJ, Nossikov A. Health interview surveys: towards international harmonization of methods and instruments. WHO Reg Publ Eur Ser. 1996;58:1-161.

7. Gomes KRO, Tanaka ACA. Morbidade referida e uso dos serviços de saúde por mulheres trabalhadoras, município de São Paulo. Rev Saude Publica. 2003;37(1):75-82. doi:10.1590/S003489102003000100012

8. Krokstad S, Kunst AE, Westin S. Trends in health inequalities by educational level in a Norwegian total population study. J Epidemiol Community Health. 2002;56(5):375-80. doi:10.1136/jech.56.5.375

9. Lepargneur $\mathrm{H}$. O novo quadro mundial da mulher. Mundo Saude. 1999;23(2):69-78.

10. Lethbridge-CeJku M, Schiller JS, Bernadel L. Summary health statistics for U.S. Adults: national health interview survey 2002. Vital Health Stat. 2004;10(222):1-151.

11. Macintyre S, Ford G, Hund K. Do women "over-report" morbidity? Men's and women's responses to structured prompting on a standard question on long standing illness. Soc Sci Med. 1999;48(1):89-98. doi:10.1016/ S0277-9536(98)00292-5
12. Macintyre S, Hunt K, Sweeting H. Gender differences in health: are things really as simple as they seem? Soc Sci Med. 1996;42(4):617-24. doi:10.1016/02779536(95)00335-5

13. Mackenbach JP, van den Bos J, Joung IM, van de Mheen $\mathrm{H}$, Stronks K. The determinants of excellent health: different from the determinants of ill-health? Inter J Epidemiol. 1994;23(6):1273-81. doi:10.1093/ ije/23.6.1273

14. National Center for Health Statistics. Health, United States, 1995. Hyattsville, Md: Public Health Service; 1996.

15. National Center for Health Statistics. Health, United States, 1998 with socioeconomic status and health chart book. Hyattsville, Md; 1999.

16. Pinheiro RS, Viscava F, Travassos C, Brito AS. Sexo, morbidade, acesso e utilização de serviços de saúde no Brasil. Cienc Saude Coletiva. 2002;7(4):687-707. doi:10.1590/S1413-81232002000400007

17. Plan and operation of the Third National Health and Nutrition Examination Survey, 1988-94. Series 1: programs and collection procedure. Vital Health Stat. 1994;1(32):1-407.

18. Restrepo HE. Epidemiologia y control del câncer de la mujer en América Latina y del Caribe. In: Gómez $E$, editor. Genero, mujer e salud en las Américas. Washington, DC: Organización Panamericana de la Salud; 1993. p.98-113. (OPS. Publicación Científica; 541).

19. Schiller JS, Bernadel L. Summary health statistics for the U.S. population: national health interview survey, 2002. Vital Health Stat. 2004;10(220):1-101.

20. Szwarcwald CL, Souza-Júnior PRB, Esteves MAP, Damacena GN, Viacava F. Socio-demographic determinants of self-rated health in Brazil. Cad Saude Publica. 2005;21(Supl 1):S54-64.

21. Verbrugge LM. The twain meet: empirical explanations of sex differences in health and mortality. J Health Soc Behav. 1989;30(3):282-304. doi:10.2307/2136961

Financiado pela Secretaria de Vigilância em Saúde/Ministério da Saúde (VIGISUS I), pelo Instituto Nacional de Câncer/ Ministério da Saúde e pelas secretarias de saúde dos estados do Rio Grande do Norte, Santa Catarina e Sergipe e da cidade de Curitiba. 